\title{
Kinetics of solid-gas reactions characterized by scanning $A C$ nano-calorimetry with application to Zr oxidation
}

\section{Citation}

Xiao, Kechao, Dongwoo Lee, and Joost J. Vlassak. 2014. "Kinetics of Solid-Gas Reactions Characterized by Scanning AC Nano-Calorimetry with Application to Zr Oxidation." Applied Physics Letters 105 (17) (October 27): 171901. Portico. doi:10.1063/1.4900779.

\section{Published Version}

doi:10.1063/1.4900779

\section{Permanent link}

http://nrs.harvard.edu/urn-3:HUL.InstRepos:22060986

\section{Terms of Use}

This article was downloaded from Harvard University's DASH repository, and is made available under the terms and conditions applicable to Open Access Policy Articles, as set forth at http:// nrs.harvard.edu/urn-3:HUL.InstRepos:dash.current.terms-of-use\#OAP

\section{Share Your Story}

The Harvard community has made this article openly available.

Please share how this access benefits you. Submit a story.

\section{Accessibility}




\title{
Kinetics of solid-gas reactions characterized by scanning AC nano-calorimetry with application to $\mathrm{Zr}$ oxidation
}

\author{
Kechao Xiao ${ }^{1}$, Dongwoo Lee ${ }^{1}$, Joost J. Vlassak ${ }^{1, \text { a) }}$ \\ ${ }^{1}$ School of Engineering and Applied Sciences, Harvard University, 29 Oxford Street, \\ Cambridge, MA 02138, USA
}

\begin{abstract}
Scanning AC nano-calorimetry is a recently developed experimental technique capable of measuring the heat capacity of thin-film samples of a material over a wide range of temperatures and heating rates. Here we describe how this technique can be used to study solid-gas phase reactions by measuring the change in heat capacity of a sample during reaction. We apply this approach to evaluate the oxidation kinetics of thin-film samples of zirconium in air. The results confirm parabolic oxidation kinetics with an activation energy of $0.59 \pm 0.03 \mathrm{eV}$. The nano-calorimetry measurements were performed using a device that contains an array of micromachined nano-calorimeter sensors in an architecture designed for combinatorial studies. We demonstrate that the oxidation kinetics can be quantified using a single sample, thus enabling high-throughput mapping of the composition-dependence of the reaction rate.
\end{abstract}

a) e-mail address: vlassak@esag.harvard.edu 
Reactions between solids and gasses play an important role in modern technology $\left.{ }^{1}\right]$. They encompass a broad field, including oxidation of metals, reduction of oxides, roasting of sulfide minerals, synthesis of thin-film coatings, and catalysis, to cite just a few examples. State-of-the-art integrated circuits would be unthinkable without the oxidation reaction to create the gate oxide or the etch processes to define patterns. Use of structural materials at elevated temperatures is often limited by their oxidation behavior, but can be extended through use of protective coatings $\left[{ }^{2-4}\right]$.

Solid-gas reactions, and high-temperature oxidation reactions in particular, are often characterized using thermogravimetric analysis (TGA). In this technique changes in the mass of the solid are measured as a function of temperature and time, and related to the extent of reaction. The technique works very well for bulk materials, but is less effective for coatings because the coating needs to be removed from the substrate to avoid the enormous dilution effect caused by substrate $\left[{ }^{5,6}\right]$. This extra step in sample preparation can be problematic and is sometimes not feasible when the sample is very thin $\left[{ }^{5}\right]$.

Here we propose the use of nano-calorimetry to characterize the oxidation behavior of very thin coatings. Nano-calorimetry is a very sensitive calorimetry technique capable of measuring the thermodynamic properties of nano-quantities of materials. The technique has been applied to study phase transformations in thin-film samples with thickness as small as a few nanometers $\left[^{7-15}\right]$. A recently developed scanning AC nano-calorimetry technique can be used to measure heat capacity over a wide range of temperatures (up to $1300 \mathrm{~K}$ ) and heating rates (from isothermal to $3000 \mathrm{~K} / \mathrm{s}$ ) $\left[{ }^{16-18}\right]$; the technique has been combined with in-situ X-ray diffraction for structural analysis of reaction products $\left[{ }^{19,}{ }^{20}\right]$. Here we describe how the technique can be used to study solid-gas phase reactions by measuring the change in heat capacity of a sample during reaction. We demonstrate this approach by evaluating the oxidation kinetics of sputter-deposited thin films of zirconium in air.

The nanocalorimetry measurements in this study were performed using a parallel nano-scanning calorimeter (PnSC) device. This device is described in detail elsewhere ${ }^{16}$ 21,22 . Briefly, it consists of a silicon substrate with a $5 \times 5$ array of independently controlled nano-calorimeter sensors as shown in Fig. 1. Each sensor contains a tungsten four-point electrical probe that serves both as a heating element and a resistance thermometer and that is fully encapsulated within a silicon nitride membrane. In a typical calorimetry measurement, a thin-film sample is deposited in the shaded area between the two sensing leads and an electric current is supplied through the tungsten heating element. The measured current and voltage are used to determine the power supplied to the sample, while the temperature of the sample is determined from the resistance of the heating element, which is calibrated to temperature $\left[{ }^{16}\right]$. The PnSC is installed in a probe card that is mounted inside a high-vacuum furnace with a base pressure of $1 \times 10^{-7}$ Torr. In this study, all measurements were performed either in vacuum or in air.

All nanocalorimetry measurements were made using a custom, low-noise data acquisition system described in detail in reference $\left[{ }^{16,19}\right]$. A carefully designed current profile, containing direct current (DC) and alternating current (AC) components, was 
of $520.83 \mathrm{~Hz}$, produced temperature oscillations of approximately $5 \mathrm{~K}$ in the sample. As described in detail elsewhere $\left[{ }^{16}\right]$, the nonlinear response of the sensors introduces harmonic components into the measured voltage, which are then isolated using harmonic analysis. By determining the amplitude $V_{2 f}$ of the $2 f$ harmonic, the heat capacity of sample and addendum can be calculated from $\left[{ }^{16}\right]$ :

$$
C=\frac{5 i^{2} I_{0} R_{0} k}{8 \pi f V_{f}}
$$

where $I_{0}$ is the DC component of the applied current, $i$ the amplitude of the AC component, $R_{0}$ the ambient-temperature resistance of the calorimeter sensor, and $k$ its temperature derivative. In the harmonic analysis, the experimental sensor voltage was partitioned into segments containing two cycles and Eq. 1 was evaluated for each segment to provide $C$ as a function of time and temperature.

After fabrication of the PnSC device, the nano-calorimetry sensors were calibrated to determine the $R_{0}$ and $k$ values [ ${ }^{16,21}$. Zirconium films with a thickness of approximately $200 \mathrm{~nm}$ were deposited using magnetron sputtering from a $\mathrm{Zr}$ (99.999\% pure) target in a DC magnetron deposition system (AJA International, North Scituate, MA) with a base pressure of $1 \times 10^{-7}$ Torr. The magnetron was powered at $80 \mathrm{~W}$ and the 43-minute deposition proceeded in an atmosphere of 5 mTorr of Ar. Zr deposition was limited to the sample area of the calorimeter sensors through use of a shadow mask. Test samples deposited under identical conditions on dummy substrates were analyzed with profilometry to determine the film thickness $h_{Z r}$.

X-ray diffraction measurements were performed on the samples before and after oxidation using a D8 Discover diffraction system (Bruker, Billerica, MA). In the as-deposited state, the Zr films had a hexagonal crystal structure with a $\{0002\}$ fiber texture, as indicated in Fig. 2 (Powder Diffraction File \#01-078-2921 ${ }^{23}$ ). Before the oxidation experiments, the $\mathrm{Zr}$ samples were heated three times to $1150 \mathrm{~K}$ and then cooled to room temperature at a rate of $700 \mathrm{~K} / \mathrm{s}$. These heating cycles were performed in vacuum using the nano-calorimeter sensors and served to measure the heat capacity of the samples as a function of temperature. The heating cycles also resulted in sharper $\mathrm{Zr}$ \{0002\} diffraction peaks (Fig. 2), as the grain size increased from $18 \mathrm{~nm}$ in as-deposited $\mathrm{Zr}$ to $33 \mathrm{~nm}$ based on a Scherrer analysis of the diffraction peaks. After thermal cycling, the $\mathrm{Zr}$ samples were oxidized by heating the nano-calorimeter sensors in air. In the XRD scans obtained after the oxidation experiments, the $Z r$ diffraction peak was replaced with the $\{-111\}$ peak of monoclinic $\mathrm{ZrO}_{2}$ (Powder Diffraction File \#01-070-2491 ${ }^{23}$ ), indicating complete oxidation of the $\mathrm{Zr}$ to $\mathrm{ZrO}_{2}$.

Isothermal oxidation experiments were performed in air on $\mathrm{Zr}$ thin-film samples, while measuring the heat capacity of the samples in situ. Because $\mathrm{ZrO}_{2}$ has a larger specific heat capacity than $\mathrm{Zr}\left[{ }^{24}\right]$, the heat capacities of the samples increase as the oxide scale grows. The increase in heat capacity of the samples, $\Delta C$, is readily measured using scanning AC nano-calorimetry. The results are plotted in Fig 3(a) as a function of time and for different temperatures. Test temperatures below $1200 \mathrm{~K}$ were selected to avoid 
change in heat capacity by

$$
\Delta h=\frac{\Delta C}{\left(C_{m, Z r O_{2}}-C_{m, Z r}\right)} \frac{V_{m, Z r O_{2}}}{S}=\lambda \Delta C,
$$

where $\Delta h$ is the change in oxide thickness during the experiment, $V_{m, Z \mathrm{ZrO} 2}$ is the molar volume of $\mathrm{ZrO}_{2}$, and $S$ represents the sample area over which the $\mathrm{Zr}$ oxidizes. In this expression, $C_{m, Z \mathrm{ZrO} 2}$ and $C_{m, Z r}$, represent the molar heat capacities of $\mathrm{ZrO}_{2}$ and $\mathrm{Zr}$, respectively. If the rate of oxidation is limited by diffusion of oxygen through the oxide layer, which is expected for our experimental conditions $\left[{ }^{25,26}\right]$, growth of the oxide layer is described by the following equation $\left[{ }^{25}\right]$ :

$$
\frac{d \Delta h}{d t}=\frac{K}{\Delta h+h_{i}}
$$

where $K$ is the parabolic rate constant and $h_{i}$ is the oxide thickness at the onset of the isothermal segment of the experiment, which consists of both the native oxide and the oxide grown during the temperature ramp that precedes the isothermal segment. $K$ is typically described by an Arrhenius relationship $\left[{ }^{26,27}\right.$ and a logarithmic graph of $K$ versus reciprocal temperature provides the activation energy $E_{a}$ for oxygen diffusion through the oxide. During isothermal oxidation, $K$ is constant and Eq. (3) is readily integrated over time. Noting that the time origin is chosen at the start of the isothermal section when $\Delta h=0$, we find

$$
\Delta h^{2}+2 \Delta h h_{i}=2 K t
$$

Equation (4) represents the well-known parabolic rate law for isothermal oxidation. During isothermal oxidation, $\lambda$ is constant and the oxide thickness scales linearly with the change in heat capacity of the sample. Combining Eqs. (2) and (4) then yields an equation that describes the evolution of the heat capacity of the sample during oxidation,

$$
\Delta C^{2}+2 \Delta C C_{i}=2 \frac{K}{\lambda^{2}} t=2 K_{c} t
$$

where $C_{i}=h_{i} / \lambda$. The parameters $C_{i}$ and $K_{c}$ can be determined from a least-squares fit of Equation (5) to the experimental $\Delta C$ - $t$ data in Fig.3. The fitting results are shown as solid lines in Fig 3(a), and the corresponding values of $C_{i}$ and $K_{c}$ are listed in Table I. Equation (5) agrees well with the experimental heat capacity curves up to approximately $80 \%$ of their maximum value, demonstrating that the reaction indeed follows the parabolic rate law and oxidation is diffusion limited. As most of the $\mathrm{Zr}$ is consumed, however, the oxidation rate decreases below the parabolic rate. This is caused by the non-uniform temperature distribution in the nano-calorimeter sensors [ $\left.{ }^{16,21,22}\right]$, which gradually lowers the average oxidation rate as the $\mathrm{Zr}$ is consumed through the thickness of the sample. The value of $\lambda$ can be calculated from the change in heat capacity of a fully oxidized 


$$
\lambda=\frac{h_{Z r O_{2}}}{\left(C_{x}\right)_{\max }+C_{i}}=\frac{R_{P B} h_{Z r}}{\left(C_{x}\right)_{\max }+C_{i}},
$$

where $h_{\mathrm{ZrO} 2}$ is the thickness of $\mathrm{ZrO}_{2}$ film and $R_{P B}$ is the Philling-Bedworth ratio for $\mathrm{Zr}$, which is taken to be $1.56\left[{ }^{28}\right]$. The value of $\lambda$ is found to be $(0.76 \pm 0.05) \mathrm{m} /(\mathrm{J} / \mathrm{K})$ for all samples. This value is larger than expected based on the sample areas of the sensors, because the temperature non-uniformity within the sensing areas decreases the area over which $Z r$ is oxidized. Once $\lambda$ is known, the parabolic rate constant $K$ is calculated from $K=\lambda^{2} K_{\mathrm{c}}$. A logarithmic graph of $K$ versus reciprocal temperature is shown in Fig. 3(b) for all isothermal measurements, together with values reported in previous works $\left[{ }^{27,29}\right]$. The value obtained in our measurements is roughly one to two orders of magnitude larger than the literature values, which may be expected because the very small grain size of our samples promotes grain boundary diffusion of oxygen through the oxide $\left[{ }^{30}\right]$. A least square line fit of the data then yields an activation energy of $E_{a}=0.60 \pm 0.09 \mathrm{eV}$ for the oxidation process, comparable to the activation energy obtained from low-temperature oxidation data in the literature $\left[{ }^{27}\right]$.

If the oxidation reaction is diffusion limited, it is also possible to quantify the reaction kinetics by performing several temperature scans on a single sample, instead of performing isothermal measurements on several different samples [ $\left.{ }^{31}\right]$. This approach is demonstrated in Fig. 4, where three different temperature scans are shown for a single Zr sample: Scan 1 was performed in vacuum, while scans 2 and 3 were performed in air. Scan 1 provides the heat capacity of the unreacted $\mathrm{Zr}$ sample as a function of temperature; scan 2 shows how the heat capacity of the sample changes as the sample oxidizes during the temperature scan; scan 3 provides the heat capacity of the fully oxidized sample. Scan 2 overlaps well with the scan 1 below $500 \mathrm{~K}$, then rises above scan 1 to join scan 3 at $810 \mathrm{~K}$. The overlap between scans 1 and 2 at low temperature indicates that the $\mathrm{Zr}$ film is well protected by its native oxide and that there is no appreciable oxide growth in this temperature range. The native oxide thickness is

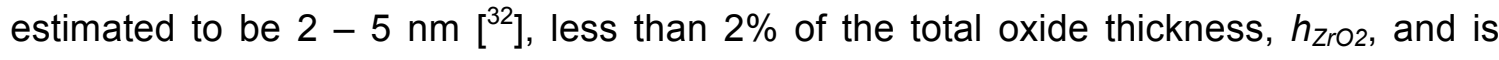
neglected in the following analysis. The overlap between scans 2 and 3 at high temperature indicates that the sample is fully oxidized at the end of the second scan, in agreement with the XRD results. The fraction of the $\mathrm{Zr}$ sample that has been oxidized in scan 1 is then calculated from

$$
\frac{\Delta h}{h_{\mathrm{ZrO}_{2}}}=\frac{C_{2}-C_{1}}{C_{3}-C_{1}},
$$

where $C_{1}, C_{2}$ and $C_{3}$ are the experimental heat capacities from scans 1 though 3 , respectively. The thickness of the oxide scale and the oxidation rate can be calculated from scan 2 using Eq. (7). The results are plotted as a function of temperature in Fig. 4(b). According to Eq. (3), the parabolic rate coefficient $K$ is given by $\Delta h \times \mathrm{d} \Delta h / \mathrm{d} t$. An Arrhenius graph of $K$ obtained from the scanning measurement is shown in Fig 3(b), along with the results from the isothermal measurements. The figure demonstrates a 
$E_{a}=0.59 \pm 0.03 \mathrm{eV}$. This agreement validates the scanning AC nano-calorimetry approach to evaluate reaction kinetics using a single sample.

While isothermal measurements may be required to establish the mechanism controlling the reaction rate, each isothermal measurement provides the rate coefficient at a single temperature. Establishing the temperature dependence of the reaction requires many measurements at different temperatures. In contrast, scanning measurements on a single sample allow direct extraction of the rate coefficient as a function of temperature, thus providing an efficient method of evaluating reaction kinetics. We anticipate that this feature will make the scanning technique an attractive choice for fast screening of materials. For instance, the oxidation resistance of an entire alloy system can be determined by performing scanning $A C$ measurements on a composition spread synthesized using magnetron sputtering - something the PnSC device is ideally suited for because of its array design [ $\left.{ }^{33-35}\right]$. More generally, one can envision using this approach to characterize a broad range of solid-gas phase reactions.

This work was supported by the Air Force Office of Scientific Research under Grant FA9550-12-1-0098. It was performed in part at the Center for Nanoscale Systems at Harvard University, which is supported by the National Science Foundation under Award ECS-0335765, and at the Materials Research Science and Engineering Center at Harvard University, which is supported by the National Science Foundation under Award No. DMR-0820484. 
Table I. Experimental parameters and fitting results

\begin{tabular}{|c|c|c|c|c|c|c|}
\hline Cell & $\begin{array}{c}T \\
(\mathrm{~K})\end{array}$ & $\begin{array}{c}h_{\mathrm{Zr}} \\
(\mathrm{nm})\end{array}$ & $\begin{array}{c}h_{\mathrm{ZrO} 2} \\
(\mathrm{~nm})\end{array}$ & $\begin{array}{c}C_{\mathrm{i}} \times 10^{8} \\
(\mathrm{~J} / \mathrm{K})\end{array}$ & $\begin{array}{c}K_{\mathrm{c}} \times 10^{15} \\
\left(\mathrm{~J}^{2} / \mathrm{K}^{2} / \mathrm{s}\right)\end{array}$ & $\begin{array}{c}K \times 10^{15} \\
\left(\mathrm{~m}^{2} / \mathrm{s}\right)\end{array}$ \\
\hline 1 & 660 & 175 & 271 & 2.0 & 0.63 & 0.37 \\
\hline 2 & 710 & 204 & 317 & 4.5 & 1.2 & 0.72 \\
\hline 3 & 760 & 202 & 313 & 2.9 & 1.9 & 1.2 \\
\hline 4 & 781 & 220 & 341 & 1.0 & 3.3 & 2.0 \\
\hline 5 & 835 & 183 & 284 & 1.4 & 5.6 & 3.3 \\
\hline 6 & $360-840$ & 211 & 327 & - & - & - \\
\hline
\end{tabular}

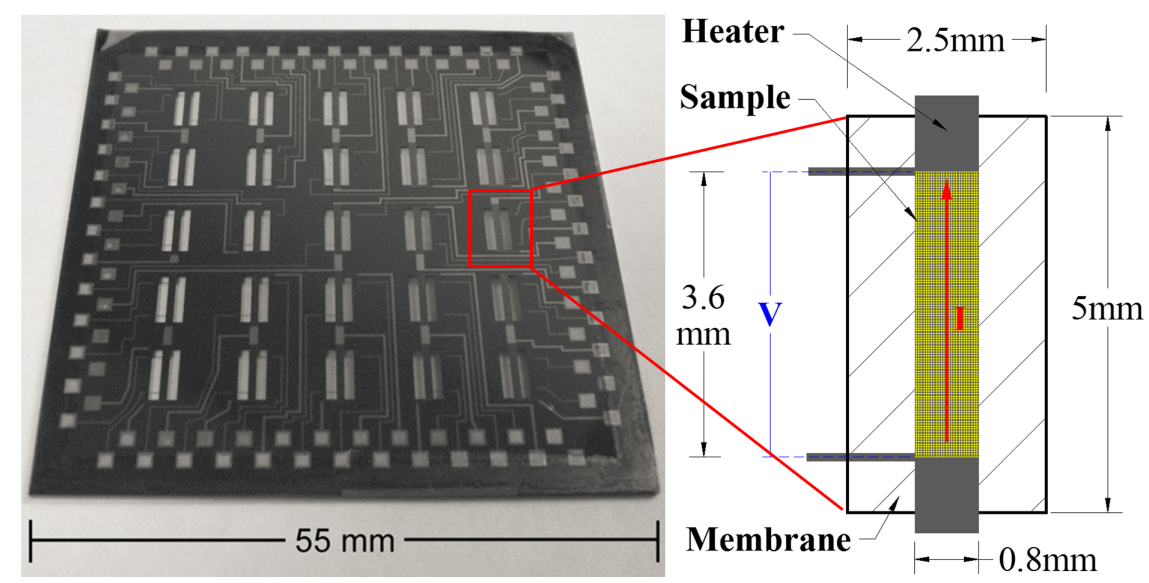

Figure 1: (left) photo of the PnSC device; (right) schematic of the calorimetry sensor.

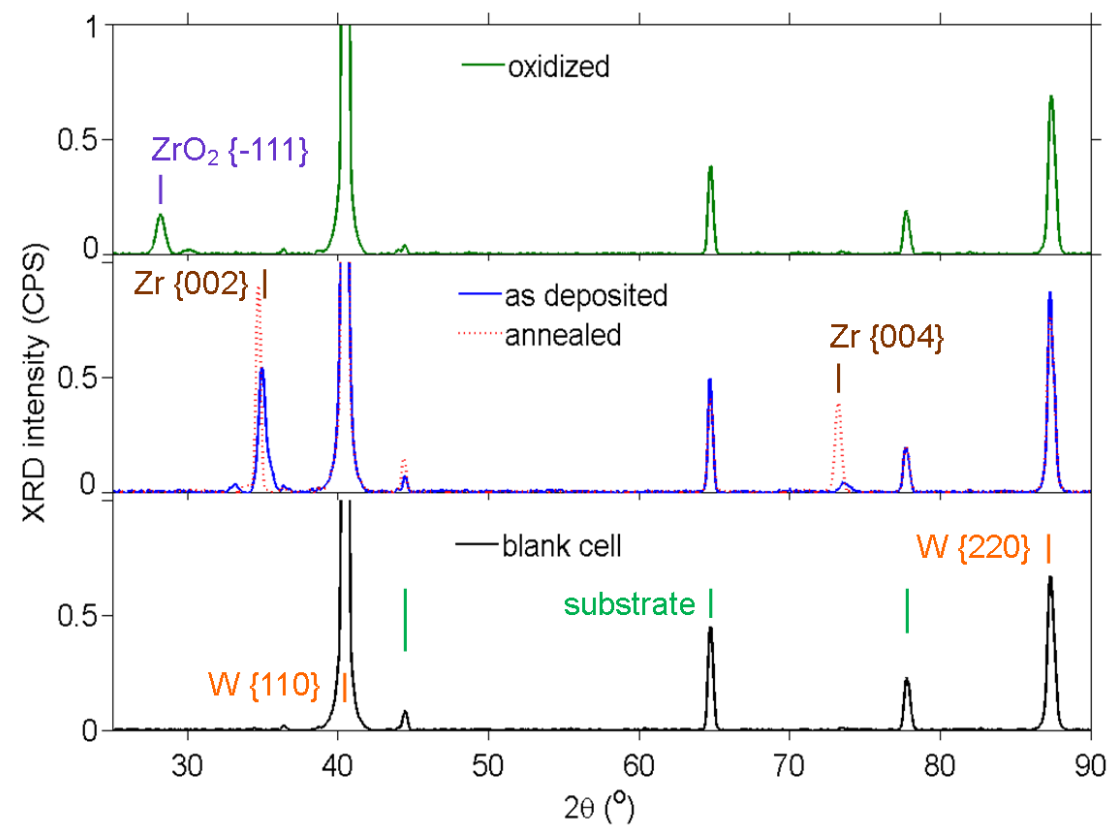


Figure 2: XRD patterns for cell with no sample, with Zr sample and with sample after oxidation experiments. The patterns were collected for $300 \mathrm{~s}$ each.
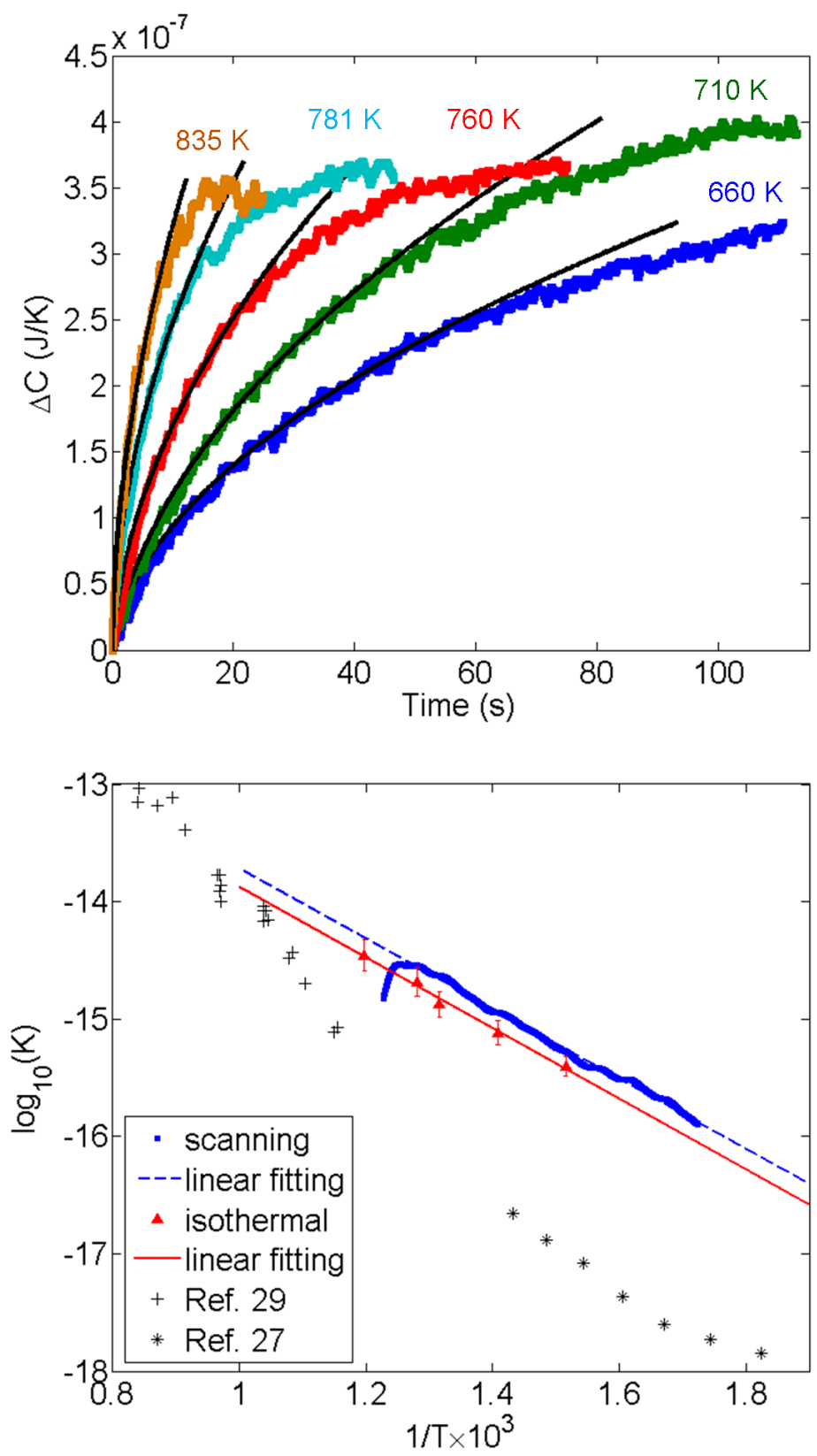

Figure 3: (top) Change in heat capacity as a function of time for five different isothermal oxidation experiments; the solid lines represent fits of Eq. (5) to the experimental data. (bottom) Logarithm of the parabolic constant $K$ as a function of reciprocal temperature for the isothermal (triangles) and scanning (squares) measurements, along with linear least squares fits and data from references [27, 29]. 


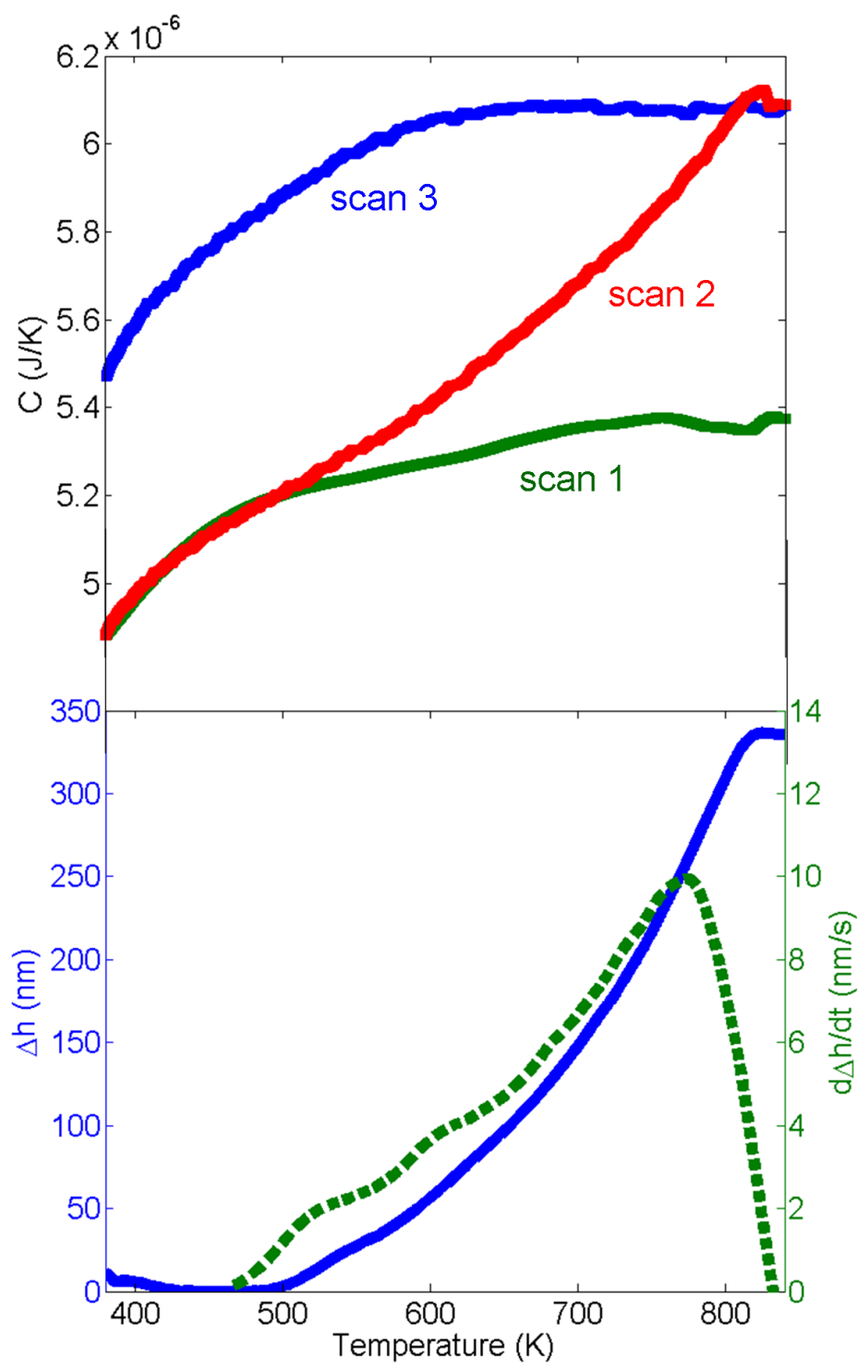

Fig. 4: (top) Results of scanning measurements in vacuum (scan 1) and air (scans 2 and 3). The scanning rate is $700 \mathrm{~K} / \mathrm{s}$ for scan 1, and $5 \mathrm{~K} / \mathrm{s}$ for scans 2 and 3 . The heat capacity of the addendum from the calorimeter is included in all three measurements. (bottom) Graphs of $\Delta \underline{h}$ and $\mathrm{d} \Delta h / \mathrm{d} t$ derived from the experimental data using Eq. (7). 


\section{References}

1. E. J. Szekely J, Sohn HY, Gas-solid reactions. (Academic Press Inc, New York, 1976).

2. G. Wranglén, An introduction to corrosion and protection of metals. (Chapman and Hall Ltd., London, 1985).

3. T. Yamashita, G. Chen, J. Shir and T. Chen, Magnetics, IEEE Transactions on 24 (6), 2629-2634 (1988).

4. A. L. Rudd, C. B. Breslin and F. Mansfeld, Corrosion Science 42 (2), 275-288 (2000).

5. M. Leskelä, T. Leskelä and L. Niinistö, Journal of thermal analysis $\mathbf{4 0}$ (3), 1077-1088 (1993).

6. J. Lin, B. Mishra, J. Moore and W. Sproul, Surface and Coatings Technology 202 (14), 3272-3283 (2008).

7. B. Yang, A. Abyzov, E. Zhuravlev, Y. Gao, J. Schmelzer and C. Schick, The Journal of chemical physics 138 (5), 054501 (2013).

8. A. A. Minakov and C. Schick, The Review of scientific instruments 78 (7), 073902 (2007).

9. E. Olson, M. Y. Efremov, M. Zhang, Z. Zhang and L. Allen, Journal of Applied Physics 97 (3), 034304 (2005).

10. P. J. McCluskey and J. J. Vlassak, Scripta Materialia 64 (3), 264-267 (2011).

11. S. Lai, J. Guo, V. Petrova, G. Ramanath and L. Allen, Physical Review Letters 77 (1), 99 (1996).

12. S. Lai, J. Carlsson and L. Allen, Applied Physics Letters 72 (9), 1098-1100 (1998).

13. M. Zhang, M. Y. Efremov, F. Schiettekatte, E. Olson, A. Kwan, S. Lai, T. Wisleder, J. Greene and L. Allen, Physical Review B 62 (15), 10548 (2000).

14. W. Chen, D. Zhou, G. Xue and C. Schick, Frontiers of Chemistry in China 4 (3), 229-248 (2009). 
16. K. Xiao, J. M. Gregoire, P. J. McCluskey and J. J. Vlassak, Review of Scientific Instruments 83 (11), 114901-114901-114915 (2012).

17. K. Xiao and J. J. Vlassak, Thermochimica Acta (2014). DOI:

10.1016/j.tca.2014.05.006

18. D. Lee, G.-D. Sim, K. Xiao, Y. S. Choi and J. J. Vlassak, Journal of Applied Physics 114 (21), 214902 (2013).

19. K. Xiao, J. M. Gregoire, P. J. McCluskey, D. Dale and J. J. Vlassak, Journal of Applied Physics 113 (24), 243501 (2013).

20. J. M. Gregoire, K. Xiao, P. J. McCluskey, D. Dale, G. Cuddalorepatta and J. J. Vlassak, Applied Physics Letters 102 (20), 201902 (2013).

21. P. J. McCluskey and J. J. Vlassak, Journal of Materials Research 25 (11), 2086-2100 (2010).

22. P. J. McCluskey and J. J. Vlassak, Thin Solid Films 518 (23), 7093-7106 (2010).

23. P. D. File, The International Centre for Diffraction Data (ICDD) 12 (2005).

24. M. W. Chase and J. A. N. A. Force, (1998).

25. B. Cox, in Advances in Corrosion Science and Technology (Springer, 1976), pp. 173-391.

26. K. R. Lawless, Reports on Progress in Physics 37 (2), 231 (1974).

27. E. A. Gulbransen and K. F. Andrew, Trans AIME 185, 515-525 (1949).

28. W. M. Haynes, CRC handbook of chemistry and physics. (CRC press, 2012).

29. D. Cubicciotti, Journal of the American Chemical Society 72 (9), 4138-4141 (1950).

30. B. Cox and J. Pemsler, Journal of Nuclear Materials 28 (1), 73-78 (1968).

31. R. Highmore, J. Evetts, A. Greer and R. Somekh, Applied Physics Letters 50 (10), 566-568 (1987).

32. U. Evans, The Corrosion and Oxidation of Metals. (Edward Arnold, London, 1960).

33. J. M. Gregoire, P. J. McCluskey, D. Dale, S. Ding, J. Schroers and J. J. Vlassak, $\sim$ * $\cdots$ r. 
34. P. J. McCluskey, K. Xiao, J. M. Gregoire, D. Dale and J. J. Vlassak, Thermochimica Acta (2014). DOI: 10.1016/j.tca.2014.07.023

35. Y. Motemani, P. J. McCluskey, C. Zhao, M. J. Tan and J. J. Vlassak, Acta materialia 59 (20), $7602-7614$ (2011). 